\title{
ON CONDITIONS OF DIFFERENTIABILITY OF LOCALLY COMPACT GROUPS
}

\author{
MASATAKE KURANISHI
}

A formulation of the differentiability of the product function of a locally compact group at the identity may be given in the following way: In a sufficiently small euclicean neighborhood of the identity product satisfies the condition :

$$
|x y-(x+y)| \leqq M(|x|+|y|)|x|
$$

where $M(\lambda)$ is a function such that $M(\lambda) \rightarrow 0$ as $\lambda \rightarrow 0$. From this we can deduce easily

$$
\lim _{n \rightarrow \infty} 2^{n}\left(\frac{1}{2^{n}} x \frac{1}{2^{n}} y\right)=x+y .
$$

In a differentiable group we can introduce a coordinates system in which it stands that $x^{m}=m x$. In this coordinates system the above equality can be written

$$
\lim _{n \rightarrow \infty}\left(x^{1 / 2^{n}} y^{1 / 2^{n}}\right)^{2^{n}}=x+y,
$$

where $x, x^{1 / 2}, x^{1 / 2^{2}}, \ldots, x^{1 / 2^{n}}, \ldots$ is the sequence of successive square roots of $x$ in the neighborhood of the identity. In words, the function sequence $F_{n}(x, y)=\left(x^{1 / 2^{n}} y^{1 / 2^{\prime \prime}}\right)^{2^{n}}$ converges to the product of an abelian local group. It is a remarkable fact that this property is independent of the choice of the coordinates system, and it will be reasonable that this is one of the topological group theoretical formulations of the differentiability of the product function at the identity.

P. A. Smith proved that under the condition (*) a euclidean local group becomes a lie group. The main purpose of this paper is to obtain conditions that a locally compact group forms a lie group from the last point of view.

Let $G$ be a locally compact group and let $U$ be a neighborhocd of the identity $e$. If, for an element $x$ of $U, x^{2}, x^{2^{n}}, x^{n^{3}}, \ldots, x^{2^{n}} \in \bar{U}, x^{2^{2+1}} \notin \bar{U}$, we put $n=\partial_{U}(x)$. If such an $n$ does not exist, we put $\delta_{U}(x)=\infty$. $G$ is said to have property $(S)$ if there exists a neighborhood $U$ of $e$ such that $\delta_{U}(x)$ is finite for every elment $x$ of $\bar{U}-\{e\}$. It will ke shown that if $G$ has property (S) we can define a function sequence $F_{n}(x, y)=\left(x^{1 / 2^{n}} y^{1 / 2^{n}}\right)^{2^{n}}$, which we shall call Lie's

Received February 28, 1950. 
function sequence, in $\Phi \times \Phi$, where $\Phi$ is a closed subset of $\bar{U}$. Theorem 1 states that if Lie's function sequence has a subsequence converging to a function, which defines product in $\Phi$ in such a way that $\Phi$ becomes an abelian local group, $G$ must be a Lie group. Theorem 2 states that if the Lie's function sequence has, roughly speaking, a uniformly converging subsequence, $G$ is a Lie group. As a corollary it is shown that a locally compact group of transformations of class $C^{1}$ which is effective in the stringent sense of S. Bochner and D. Montgomery ") must be a Lie group.

\section{Conditions of differentiability}

In this section a locally compact separable group $G$ having property $(S)$ will be treated. Take a neighborhood $U=U^{-1}$ of $e$ with $\bar{U}$ compact and such that $\delta_{U}(x)$ is finite for $x \in \bar{U}-\{e\}$ and such that in $U^{3}$ square root is unique, that is, from $x^{2}=y^{2} \in U^{3}$ and $x, y \in U^{3}$ follows $x=y .{ }^{2)} \quad$ Such a neighborhood is said to be regular. Put

$$
\begin{aligned}
& \Phi_{n} \bar{U}=\left\{x \mid \text { there exists } x^{1 / 2^{k}} \in \bar{U} \text { such that }\left(x^{1 / 2^{k}}\right)^{2^{k}}=x \text { for every } k \leqq n\right. \text { and } \\
& \left.\left(x^{1 / 2^{k}}\right)^{2^{h}} \subset \bar{U} \text { for } h \leqq k\right\}, \Phi^{\bar{\sigma}}=\bigcap_{n=1}^{\infty} \Phi_{n}^{\bar{U}} \text {. }
\end{aligned}
$$

$\Phi_{n} \tilde{U}$ and $\Phi^{\bar{C}}$ are closed sets. It is easy to see that $\left(x^{1 / 2^{n}}\right)^{2^{m}}=x^{1 / 2^{n-m}}(n>m)$. An element $(\neq e)$ of $\Phi^{\vec{U}}$ is called regular (in $\left.\bar{U}\right)$. For a regular element $x$ we mean by the square root sequence of $x$ the sequence $\left\{x^{1 / 2^{n}}\right\}_{n=0,1,2, \ldots}$. If $y \neq e$ were a limit point of the square root sequence of $x$, then $y^{2^{k}}$ would be also limit point of $\left\{x^{1 / 2^{n}}\right\}$, hence belong to $\bar{U}$, which contradicts to property (S). Therefore the square root sequence converges to $e$. We now introduce a function on $\Phi_{n}^{\bar{C}} \times \Phi_{n}^{\vec{U}}$ by

$$
F_{n}(x, y)=\left(x^{1 / 2^{n}} y^{1 / 2^{n}}\right)^{2^{n}} \text {. }
$$

Since square root is unique and $\bar{U}$ is compact, the function can be defined and is continuous. The sequence of functions $F_{n}(x, y),(n=1,2, \ldots)$ is called Lie's function sequence of $G$ in $\bar{U}$.

Lemma 1. For every regular element $x$ of $\bar{U}$, there exists a unique one-parameter subgroup $g_{x}(\lambda)$ such that $g_{x}\left(1 / 2^{n}\right)=x^{1 / 2^{n}}$ for $n=0,1,2, \ldots$

$$
\text { Proof. If } n>m, x^{1 / 2^{n}} x^{1 / 2^{m}}=x^{1 / 2^{n}}\left(x^{1 / 2^{n}}\right)^{2^{n-m}}=\left(x^{1 / 2^{k}}\right)^{2^{n-m}} x^{1 / 2^{n}}=x^{1 / 2^{m}} x^{1 / 2^{n}} \text {. }
$$

Hence the closed subgroup $A$ generated by the square root sequence of $x$ is abelian. Since $A$ does not have arbitrarily small subgroups, $A$ is a lie group. Let $A^{0}$ be the component of $e$ in $A$. Since $A^{0}$ covers a neighborhood of $e$ and $x^{1 / 2^{n}}$ coverges to $e$, there exists an integer $k$ such that for every $m \geqslant k, x^{1 / 2^{m}} \in A^{0}$.

1) Cf. [6].

$\therefore$ Cf. Lemma 1 of [4]. 
Hence $x^{1 / \alpha^{17}} \in A^{n}$ for every $n$ and so $A=A^{0}$. Therefore for every element $y \in A$ there exist unique one-parameter subgroup $g_{y}(\lambda)$ such that $g_{y}(1)=y$. Since $A$ is a Lie group, there exists a neighborhood $W$ of $e$ such that $g_{y}(\lambda) \subset U$ for $|\lambda|$ $\leqq 1$ and for $y \in W$. If $x^{1 / 1 / k} \in W$, it follows that $g_{x^{1 / 2 k}}\left(1 / 2^{n}\right)=x^{1 / 2^{n+k}}$ and $g_{x^{1 / \Omega k}}\left(2^{k} \lambda\right)=g_{x}(\lambda)$. From this, it follows that $g_{x}\left(1 / 2^{n}\right)=x^{1 / 2^{n}}$ for every $n$.

Remark. Since no confusion will occur, we shall write $x^{\lambda}$ instead of $g_{r}(\lambda)$ in the following.

Lemma 2. There exists a real continuous function $f(x)$ defined on $\bar{U}$ satisfying the following conditions:

$$
f\left(x^{2}\right) \geqq 2 f(x) \text { for } x, x^{2} \in \bar{U}
$$

$$
f(x)=0 \text { if and only if } x=e \text {. }
$$

Proof. Put $\psi(x)=x^{2}$ for $x \in U^{2}$. Since $\psi$ is continuous and square root is unique in $U^{3}$ there exist with given $p(\neq e) \in \bar{U}$ open sets $V_{k^{(1)}} p^{\mathrm{a}^{k}}$ and $V_{k^{(2)}} p^{2^{k}}$ for $k=0,1, \ldots, \delta_{L^{2}}(p)$ such that

$$
\begin{aligned}
& \overline{V_{k}^{(1)} p^{\mathrm{2}^{k}}} \subset V_{k}^{(2)} p^{2^{k}} \subset U^{3}, \\
& \psi\left(V_{k}^{(2)} p^{2^{k}}\right) \subset V_{k+1}^{1)} p^{2^{k+1}}, \\
& V_{k}^{(2)} p^{2^{k}} \cap V_{j}^{(2)} p^{2^{j}}=\phi \quad(k \neq j),
\end{aligned}
$$

$\phi$ denoting the empty set. Construct continuous functions $f_{k}^{(p)}(x)$ for $k=0,1$, $\ldots, \delta_{U^{*}}(\not)$ defined on $U^{3}$ satisfying the conditions

$$
\begin{aligned}
& f_{k}^{(p)}(x)=\frac{1}{2^{(o) 2(p)-k},} \text { for } x \in V_{k}^{1 !} p^{p^{k}}, \\
& f_{k}^{(p)}(x)=0 \quad \text { for } x \notin V_{k}^{(2)} p^{2^{k}}, \\
& 0 \leqq f_{k}^{(p)}(x) \leqq \frac{1}{2^{\left(\delta C^{(2)}(p)-k\right)}} .
\end{aligned}
$$

Let $f^{(p)}(x)=\sum_{k=0}^{\delta_{L=0}(p)} f_{k}^{(p)}(x)$. Then it is easily shown that $f^{(p)}(x)$ satisfies the condition i). Since $\bar{U}-\{e\} \subseteq \bigcup_{\nu \in T^{-}-\{e\}} V_{0}^{(1)} p^{3},{ }^{3 !}$ there exists a countable set $\left\{p_{n}\right\}$, $(n=1,2, \ldots)$, such that $\bar{U}-\{e\} \subseteq \bigcup_{n=1}^{\infty} V_{0}^{(1)} p_{n} . \quad$ Define $f(x)=\sum_{n=1}^{\infty} \frac{1}{2^{n}} f^{\left(p_{n}\right)}(x)$, then $f(x) \mid \bar{U}$ fulfills our requirements.

The following two lemmas show the non-triviality of $\Phi^{\vec{U}}$.

Lemma 3. $\mathscr{D}^{\vec{\imath}}-\{e\} \neq \phi .^{4)}$

Levma 4. Let $Z$ be the center of $G$. If $G \cap U \supsetneq Z \cap U$, there exists a neighborhood $V$ of $e$ such that $Z \cap V \nsubseteq \Phi^{\vec{U}} \cap V$.

3) $V_{0}^{1}$ is depend with $p$.

4) Cf. Lemma 5 of [7]. 
Proof. Since $Z$ does not contain arbitrarily small subgroups, $Z$ is a Lie group. Hence there exists a discrete subgroup $H$ of $Z$ such that $Z / H$ is a torus group. $G / H$ is locally isomorphic to $G$ and the center of $G / H$ is $Z / H$. Hence we have only to treat the case where $Z$ is a torus group. Moreover, to prove the Lemma it is sufficient to prove that $G / Z$ contains at least one-parameter subgroup, ${ }^{5)}$ and for this last purpose it suffices to prove that $G / Z$ does not contain arbitrarily small subgroups. $\left.{ }^{6}\right)$

Let $\varphi$ be a natural mapping $G \rightarrow G / Z$, and put $U_{1}^{*}=\varphi\left(U_{1}\right)$, where $U_{\mathrm{k}}$ $=\{x \mid f(x)<\delta\} \subset U$ with a function $f(x)$ in Lemma 2 and a sufficiently small positive number $\delta$. Suppose $x^{*}=y^{*}$ for $x^{*}, y^{*} \in U_{1}^{*}$. Take $x, y \in U_{1}$ such that $x^{*}=\varphi(x)$ and $y^{*}=\varphi(y)$. Then $x^{2}=y^{2} z$ and $z \in U_{1}^{4} \cap Z . \quad\left(y z^{3 / 2}\right)^{2}=x^{2}$. Hence if $U_{1}$ is sufficiently small, $y z^{\mathrm{I} / 2}=x$, and so $x^{*}=y^{*}$. Thus square root is unique in $U_{1}^{*}$.

Now let $K^{*}$ be a closed subgroup contained in $U_{1}^{*} . \quad K=\varphi^{-1}\left(K^{*}\right)$ is a compact subgroup of $G$, and so $K$ is a Lie group. Hence $K^{*}$ is also a Lie group. If $K^{*}$ were not discrete, there would exist an element $k_{0}^{*}\left(\neq e^{*}\right) \in E K^{*}$ such that $k_{0}^{* 2}=e^{*}$, which is a contradiction to the fact that square root is unique in $U_{1}^{*}$. Therefore $K^{*}$ must be a finite group.

Suppose $K^{*}$ is not trivial. Take $k^{*}=\varphi(k) \neq e^{*}\left(k \in U_{1}\right)$ of $K^{*}$. We assert that $k$ is a regular element in $U$ and $\varphi\left(k^{1 / 2^{n}}\right) \in K^{*}$ for every $n$. Namely, since $K^{*}$ is finite, there exist $m$ and $l(m>l)$ such that $k^{* 2^{m}}=k^{* 2^{l}}$, and so $k^{*}=k^{* 2^{m-l}}$ $=\left(k^{* 2^{m-l-1}}\right)^{2}$. Hence $k z=h^{2}$, where $h \in U_{1}$ and $\varphi(h)=k^{* 2^{2 n-l-1}}$. Then $z \in U_{1}^{3}$ $\cap Z$ and $\left(h z^{-(1 / 2)}\right)^{2}=k$. Therefore if $U_{1}$ is sufficiently small $h z^{-(1 / 2)}=k^{1 / 2} \in U$. But as $U_{1}=\{x \mid f(x)<\delta\}$ and $f\left(x^{2}\right) \equiv 2 f(x), k^{1 / 2} \in U_{1}$. Moreover $\varphi\left(k^{1 / 2}\right)$ $=\varphi\left(h z^{-(1 / 2)}\right)=k^{* 2^{m-l-1}} \in K^{*}$. Since $b^{1 / 2} \notin Z$, we can easily prove the assertion by induction. Since $K^{*}$ is discrete, there exists a neighborhood $U_{2}^{*}$ such that $U_{:^{*}}^{*} \cap K^{*}=\left\{e^{*}\right\}$. Because $k^{1 / 2^{2}} \rightarrow e$ as $n \rightarrow \infty$, there exists an integer $n_{0}$ such that $k^{1 / 2^{n_{0}}} \in \varphi^{-1}\left(U_{2}^{*}\right)$. Hence $\varphi\left(k^{1 / 2^{n_{0}}}\right) \in U_{2}^{*} \cap K^{*}:=\left\{e^{*}\right\}$, and so $k^{1 / 2^{n_{0}}} \in Z$. Therefore $k \in Z$, which is a contradiction to $\varphi(k)=k^{*} \neq e^{*}$. Thus $K^{*}$ is trivial, and proof of our Lemma is completed.

Lemma 5. Let $\Phi$ be a locally compact abelian local group containing no subgroup in the large. Suppose for every element $x$ of $\Phi$ square root $x^{\mathrm{i} / 2}$ of $x$ exists uniquely and detemined continuously. Let $D_{1}$ be the set of element $x$ such that there exists one-parameter subgroup $x^{\lambda}(|\lambda| \leqq 1)$. Then $D_{1}$ is a closed local subgroup of $\Phi$ and is locally ismorphic to a neighborhood of the identity

5; If $H$ is a normal subgroup of $G$ and both $C / H$ and $H$ are Lie groups, then $G$ is a lie group. Cf. [2] or Theorem 7 of [3].

6) Cf. Lemma 5 of [7]. 
of a vector group, (i.e. $\Phi_{1}$ is a local vector group).

Proof. Let $\circ$ denote the product operation in $\mathscr{0} . x^{\lambda}$ is a local 1-dimensional vector group. Assume that there exist $x_{1}, x_{2}, \ldots, x_{n}$ such that for a fixed positive $\varepsilon x_{i}{ }^{\lambda}(\lambda \neq 0) \notin E_{i-1}^{(\varepsilon)}=\left\{y\left|y=x_{1}^{\lambda} \circ x_{2}^{\lambda \lambda_{0}} \ldots \circ x_{i-1}^{\lambda_{i-1}},\right| \lambda_{j} \mid<\varepsilon\right\} \subset \Phi_{1},(i=1,2$, $\ldots, n) . E_{n}^{(8)}$ is a local $n$-dimensional vector group. Suppose $E_{n}^{(\varepsilon)}$ does not cover a open set of $\mathscr{D}_{1}$. We shall show that there exists $x_{n+1} \in \Phi_{1}$ such that $x_{n+1}^{\lambda}(\lambda \neq 0) \notin E_{n}^{(\varepsilon)}$. If not, there exists a $\lambda^{\prime}$ and a sequence $x_{\nu}\left(E \mathscr{D}_{1}-E_{n}^{(\ell)}\right) \rightarrow e$ such that $x_{\nu}^{\lambda_{\nu}} \equiv x_{n}^{\xi / 2} \bmod E_{n-1}^{(\varepsilon / 2)}$ e.g. for some $\left|\lambda_{2}\right|<1$ and such that $\lambda_{\nu} \rightarrow \lambda^{\prime}$. Take a rational number $\eta(<1)$ such that $\left|\lambda^{\prime}-\eta\right|<\varepsilon_{1}^{\prime}$. Then $x_{\nu}^{\eta} \equiv x_{n}^{\eta_{\nu}} \bmod E_{n-1}^{(s,}$, where $\left|\eta_{\nu}\right|>\varepsilon / 4$. Since $x^{1 / 2}$ is a continuous function of $x, x^{\eta}$ is also, whence $x_{\nu}^{\eta} \rightarrow e$. But $x_{n}^{\eta}$ can not converge to any element of $E_{n-1}^{(\varepsilon)}$. Hence there exists $x_{n+1}$ such that $x_{n+1}^{\lambda} \notin E_{n}^{(\varepsilon)}$. Then we can easily construct $E_{n+1}^{\left(\varepsilon^{\prime}\right)}$ for a sufficiently small $\varepsilon^{\prime}>0$.

Thus to prove the lemma we have only to show that $\mathscr{D}_{1}$ is finite dimensional. Suppose $\mathscr{D}_{1}$ is infinite dimensional. Then there exists, by the above observation, a sequence of local vector group $E_{1}, E_{2}, \ldots, E_{n}, \ldots$, where $E_{n}$ is $n$-dimensional local vector group and a neighborhood of the identity of $E_{n}$ is a local subgroup of $E_{n+1}$. Let $E_{n}^{\prime}$ be a sequence of $n$-dimensional vector group such that $E_{1}^{\prime} \subset E_{!}^{\prime}$ $C \ldots \subset E_{n}{ }^{\prime} \subset E_{n+1}^{\prime} \subset \ldots$ and such that $E_{j}(j \leqq n)$ is a local subgroup of $E_{n}{ }^{\prime}$. Denote $E=\bigcup_{n=1}^{\infty} E_{n}$ and $E^{\prime}=\bigcup_{n=1}^{\infty} E_{n}{ }^{\prime}$. Consider $E^{\prime}$ as an abstract group and introduce a topology by taking $E \cap V_{v}$ as a fundamental neighborhood system of the identity in $E^{\prime}$, where $V_{\nu}$ is a fundamental neighborhood system of the identity in $\not$. We can complete $E^{\prime}$ to a group $\widetilde{E}^{\prime 7)}$ Completed neighborhood of $E \cap V$, must be isomorphic to $E \cap V_{v}$ (closure in $\mathscr{D}$ ), because of the uniqueness of completion.s' Thus $\widetilde{E}^{\prime}$ is locally compact and contains no small subgroups. Hence $\widetilde{E}^{\prime}$ is finite dimensional, which is a contradiction.

THEOREM 1. Let $G$ be a locally compact separable group having property (S) and let $U$ be a regular neighborhood. Sippose that Lie's function sequence in $\bar{U}$ has a subsequence $F_{n_{i}}(x, y)$ satisfying the following conditions:

(A) $F_{n_{i}}(x, y) \mid \emptyset^{\vec{C}}$ converges to a function $F(x, y)$ such that there exists a neighborhood $W$ of $e$ so as $F(x, y) \in \Phi^{\vec{U}}$ for $x, y \in W \cap D^{\bar{v}}$.

(B) By the product operation $x \circ y=F(x, y)$, $\phi^{\vec{u}}$ becomes an abelian local group. Then $G$ is a Lie group.

7) Theorem II of [10].

8) Theorem II of [10]. Consult also the method of completion. 
Proof. Observing that $x \circ y=x \cdot y$ when $x$ and $y$ are commutative, we can see easily that $\Phi^{\bar{U}}$ is a local group satisfying the condition of Lemma 5 , whence $\emptyset_{1}^{\bar{C}}=\left\{x \mid x^{\lambda} \in \Phi^{\tilde{U}}\right.$ for $\left.|\lambda| \leqq 1\right\}=\left\{x \mid x^{\lambda} \in \bar{U}\right.$ for $\left.|\lambda| \leqq 1\right\}$ is a local vector group. As the square root is unique in $U^{3}, F_{n}\left(p^{-1} x p, p^{-1} y p\right)=p^{-1} F_{n}(x, y) p$ for $x, y \in \Phi_{n} \bar{U}$ and $p \in U$. Hence $\left(p^{-1} x p\right) \circ\left(p^{-1} y p\right)=p^{-1}(x \circ y) p$. For a given element $p$ of $\bar{U}$, there exists $V$ such that $p^{-1} V p \subset \bar{U}$. Since $\Phi_{1}^{\vec{U}}$ is a local vector group, there exists $V_{1}$ such that for every $x \in \Phi_{1}{ }^{\bar{C}} \cap V_{1}$ it stands that $x^{\lambda} \in V$. Whence $p^{-1}\left(\mathscr{\Phi}_{1}^{\bar{C}} \cap V_{1}\right) p \subset \mathscr{\Phi}_{1}^{\bar{U}}$. Therefore the transformation $x \rightarrow p^{-1} x p$ for $p \in \bar{U}$ is an automorphism of the local vector group $\mathscr{D}_{1}^{\bar{U}}$, and so is a linear transformation. Let $A_{p}$ be the matrix of this linear transformation.

It is clear that for a sufficiently small neighborhood $L$ of $e$ in $G, L \ni p \rightarrow A_{p}$ is a representation of our group. We call this the canonical representation. Let $L^{*}$ be its kernel and $G^{*}$ be the closed subgroup generated by $L^{*}$.

$$
G^{*} \cap L=L^{*}
$$

For, take $x \in G^{*} \cap L$. Since $G^{*}$ is generated by the elements commutative with $\mathscr{\Phi}_{1}^{\vec{C}}, x$ is also commutative with $\Phi_{1}{ }^{\vec{C}}$. Hence $x \in L^{*}$ by definition.

ii)

If $G=G^{*}$, then $L$ is abelian.

Suppose $L$ is not abelian, and let $Z$ be the center of $G . \quad L \supsetneq Z \cap L$. By Lemma 4 , there exists at least one regular $x$ which is not contained in $Z$, hence at least one element $x_{1}$ of $\Phi_{1}^{\bar{C}}$ such that $x_{1} \notin Z$. Moreover $G\left(=G^{*}\right)$ is generated by $L$. Whence there is at least one element of $L$ not commutative with $x_{1}$. Therefore $G \supsetneq G^{*}$ by i).

$G^{*}$ also satisfies, as a closed subgroup of $G$, the conditions of Theorem 1 with $U \cap G^{*}$ instead of $U$. Therefore we can define $\left(G^{*}\right)^{*}$.

iii)

$$
\left(G^{*}\right)^{*}=G^{*} \text {. }
$$

Since $G^{*} \supseteq\left(G^{*}\right)^{*}$, we have only to prove that $\left(G^{*}\right)^{*} \supseteqq G^{*}$. Let $\mathscr{D}^{\prime}$ be the set of regular elements of $G^{*}$ in $G^{*} \cap U$. Then $\mathscr{D}^{\prime} \subset \Phi^{\top}$. Every element of $G^{*}$ is commutative with $\Phi^{T}$, whence with $\Phi^{\prime}$. Hence $\left(G^{*}\right) \subseteq\left(G^{*}\right)^{*}$.

Now we can complete our proof. $G / G^{*}$ is a Lie group and by ii) and iii) $G^{*}$ is an abelian normal subgroup of $G$ and is a Lie group. Hence $G$ is a Lie group by the extension theorem of Lie groups."

TheOREM 2. Let $G$ be a locally compact separable group having property (S) and let $U$ be a regular neighborhood of $G$. Suppose Lie's function sequence in $\vec{U}$ has a subsequence $F_{n_{i}}(x, y)$ satisfies the following conditions:

(C) For every neighborhood $V$ of $e$, there exists an integer $I$ such that for

9) Cf. [2] or Theorem 7 of [3]. 
every $i>I$ and $x, y \in \mathscr{D}_{n_{i}}^{\bar{e}}$

$$
V F_{n_{I}}(x, y) \exists F_{n_{\iota}}(x, y) \text {. }
$$

(D) There exists a neighborhood $W$ of e such that for $x, y \in \emptyset^{\bar{y}} \cap W$

$$
F_{n}(x, y) \subset U, \quad(n=0,1,2, \ldots)
$$

Then $G$ is a Lie group.

Proof. By (C) $F_{n_{i}}(x, y) \mid \Phi^{\imath}$ converges uniformly to a continuous function $F(x, y)$. We show that $F(x, y)$ satisfies the condition (A) and (B) of Theorem 1 .

(A) By Lemma 2, there exists a neighborhood $W_{1}$ of $e$ such that if $x \in W_{1}$, $x^{1 / 2^{n}} \in W$ for $n=1,2, \ldots$ If $x, y \in \Phi^{\vec{c}} \cap W_{1}$

$$
\left(x^{1 / 2^{n}{ }^{2} i} y^{1 / 2^{n_{i}}}\right)^{2^{n_{i}-k}}=\left(\left(x^{\mathrm{i} / 2^{k}}\right)^{1 / 2^{n_{i}-k}} \cdot\left(y^{1 / 2^{k}}\right)^{1 / 2^{n_{i}-k}}\right)^{2^{2_{i}-k}}=F_{n_{i}-k}\left(x^{1 / 2^{k}}, y^{1 / 2^{k}}\right) \in \bar{U} .
$$

Now, take $x, y$ in our $W_{1}$ thus obtained. If $v$ is a limit point of $\left(x^{1 / \Sigma^{n_{i}}} y^{1 / 2^{n_{i}}}\right)^{2^{1 n_{i}-k}}$, $\left(n_{i}>k\right)$, then $v \in \bar{U}$ and $v^{2^{k}}=F(x, y)$. Hence, for $x, y \in W_{1} \cap \emptyset^{\vec{c}}, F(x, y) \in \mathscr{D}^{-}$.

(B) $\quad \alpha) \quad(x \circ y) \circ w=x \circ(y \circ w) \quad$ (associative law)

For every neighborhood $V$ of $e$, there exists $l$ such that if $x, y \in \mathscr{D}^{\vec{c}}$

$$
V \cdot(x \circ y) \ni F_{n_{I}}(x, y),
$$

and such that if $i \geqq I$ and $x, y \in \Phi_{n_{i}}^{\bar{c}}$,

$$
V F_{n_{I}}(x, y) \ni F_{n_{i}}(x, y) \text {. }
$$

Since ${\Phi_{n}}^{\vec{\imath}}$ is closed, $F_{n}(x, y)$ is uniformly continuous, and so there exists, by (7), a neighborhood $V_{1}$ of $e$ such that for every $x, y \in \Phi^{\vec{c}}$,

$$
V^{2} \cdot(x \circ y) \ni F_{n_{I}}\left(V_{1} x \cap \mathscr{\omega}_{n_{1}}^{\vec{c}}, y\right)
$$

By $\mathscr{\Phi}_{n_{I}}^{\bar{U}} \supset \Phi_{n_{i}}^{\vec{U}},(8)$, and (9)

$$
F_{n_{i}}\left(V_{1} x \cap \Phi_{n_{i}}^{\bar{c}}, y\right) \subset V F_{n_{1}}\left(V_{1} x \cap \Phi_{n_{1}}^{\bar{c}}, y\right) \subset V^{3}(x \circ y)
$$

for every $x, y \in \Phi^{\bar{c}}$ and $i \geqq I$. Hence

$$
V^{s}((x \circ y) \supset w) \supset F_{n_{i}}\left(V_{1}(x \supset y) \cap \Phi_{n_{i}}^{\bar{c}}, w\right)
$$

If $n_{i}$ is large enough,

$$
V_{1}(x \circ y) \cap \Phi_{n_{i}}^{\bar{c}} \ni F_{n_{i}}(x, y)
$$

Therefore, by (10) and (11),

$$
V^{3}((x \circ y) \circ w) \supset F_{n_{i}}\left(F_{n_{i}}(x, y), w\right)=\left(x^{1,2^{n_{i}}} y^{1 / 2^{n_{i}}} w^{1 / 2^{n_{i}}}\right)^{2^{n_{i}}},
$$

whence

$$
(x \circ y) \circ w=\lim _{n \rightarrow \infty}\left(x^{1 / 2^{n_{i}}} y^{1 / 2^{n_{i}}} w^{1 / 2^{n_{i}}}\right)^{2^{n_{i}}} .
$$

Similarly we can obtain

$$
x \circ(y \circ w)=\lim _{n \rightarrow \infty}\left(x^{1 / 2^{n_{i}}} y^{1 / 2^{n_{i}}} w^{1 / 2^{n_{i}}}\right)^{2^{n_{i}}} .
$$


$\beta)$

$$
x \circ y=y \circ x, \quad x \circ x^{-1}=e .
$$

Since $F_{n}(x, y)=x^{3 / 2^{n}}\left(y^{3 / 2^{n}} x^{3 / 2^{n}}\right)^{2^{n}} x^{-1 / 2^{n}}=x^{1 / 2^{n}} F_{n}(y, x) x^{-1 / 2^{n}}$ and $x^{1 / 2^{n}} \rightarrow e$. Thus, by the product operation $\circ, \mathscr{D}^{\vec{V}}$ becomes an abelian local group.

Remark. If $F_{n}(x, y)$ is a sequence satisfying the condition (C), $G$ is a Lie group. For $F(x, y) \mid \Phi^{\vec{U}}$ converges uniformly to $F(x, y)$, there exists $W$ satisfying (D).

\section{Groups satisfying some metric conditions}

TheOREM 3. ${ }^{10)}$ Let $G$ be a locally compact group. Assume that for a sufficiently small neighborhood $\bar{V}$ of $e$, we can introduce a metric $\rho$ satisfying the following conditions :

(E) If $x, y, x y \in \bar{V}$, then

$$
K_{\imath} \rho(y, e) \leqq \rho(x y, x) \leqq K_{1} \cdot \rho(y, e) ;
$$

(F) If $n \leqq \delta_{V}(x), \delta_{V}(y)$, then

$$
K_{4} 2^{n} \rho(x, y) \leqq \rho\left(x^{2^{n}}, y^{2^{2 n}}\right) \leqq K_{3} 2^{n} \rho(x, y) ;
$$

with positive constante $K_{i}, i=1,2,3,4$.

Then $G$ is a Lie group.

Outline of the proof. From (F), $G$ has property (S). We shall show that $G$ satisfies the condition of Theorem 2 .

From (E) and (F) we can deduce that ${ }^{\text {in) }}$

$$
\rho\left(F_{n}(x, y), F_{n}\left(x^{\prime}, y^{\prime}\right)\right) \leqq K\left\{\rho\left(x, x^{\prime}\right)+\rho\left(y \cdot y^{\prime}\right)\right\}
$$

with a positive constant $K$.

Therefore, if we take a sufficiently small neighborhood $U$ of $e$ which is regular, the function sequence $\bar{F}_{n}(x, y) \mid \Phi^{\vec{c}} \times \emptyset^{\vec{C}}$ is a uniformily bounded and equicontinuous sequence with respect to the uniform structure introduced by $\rho$. We

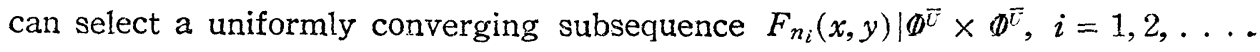
For every positive $\varepsilon$ there exists an integer $I_{1}$ such that for every $i, j \equiv I_{1}$ and $x^{\prime}, y^{\prime} \in \bar{\emptyset}^{\bar{v}}$

$$
\rho\left(F_{n_{i}}\left(x^{\prime}, y^{\prime}\right), F_{n_{j}}\left(x^{\prime}, y^{\prime}\right)\right)<\varepsilon / 3 .
$$

Since $\mathscr{D}^{\vec{U}}=\bigcap_{n=1}^{\infty} \Phi_{n}^{\vec{U}}$, there exists $I_{2}$ such that $\left.U_{\varepsilon /\left(6 K^{\prime}\right)}\left(\Phi^{\vec{U}}\right)\right) \supset \Phi_{n_{i}}^{\vec{U}}$ for every $i \geqq I_{2,}$ where $U_{\varepsilon}(\mathscr{D})$ denotes the $\varepsilon$-neighborhood of $\mathscr{D}$. Therefore, there are, for every $x, y \in \Phi_{n_{i}}^{\bar{v}}, x^{\prime}, y^{\prime} \in \Phi^{\bar{v}}$ such that

$$
\rho\left(x, x^{\prime}\right)<\varepsilon /(6 k), \quad \rho\left(y, y^{\prime}\right)<\varepsilon /(6 k) .
$$

\footnotetext{
10) This Theorem generalizes the theorem of [4].

11) Cf. $\$ 2$ of [4].
} 
From (12) and (14),

$$
\rho\left(F_{n_{i}}(x, y), F_{n_{i}}\left(x^{\prime}, y^{\prime}\right)\right)<K\left(\rho\left(x, x^{\prime}\right)+\rho\left(y, y^{\prime}\right)\right)<\varepsilon / 3 .
$$

If $I=\max \left(I_{1}, I_{2}\right)$ and $i \geqslant I$,

$$
U_{\varepsilon}\left(F_{n_{I}}(x, y)\right) \ni F_{n_{i}}(x, y)
$$

by (13), (15), (15) $i$.

As the uniform structure of the compact space is unique, we can deduce from (16) that the condition (C) of Theorem 2 is satisfied. (D) follows immediately from (12) putting $x^{\prime}=y^{\prime}=e$.

\section{Groups of transformation of class $C^{1}$}

Let $G$ be, throughout this section, a locally compact group of transformation of class $C^{1}$ of a manifold $M$ of class $C^{1{ }^{12}} \quad$ Elemants of $G$ are denoted by $v, w$, $x, y$, etc. and point of $M$ by $p, q, r, s$, etc. The point of $M$ to which $p$ is transformed by $x$, will be denoted by $x(p)$ or $f(x, p)$. Let a coordinate neighborhood $Q$ of $M$ with $\bar{Q}$ compact be fixed. The $i$-coordinates of $p, x(p)$, and $f(x, p) \in Q$ are denoted by $\dot{p}_{i}, x_{i}(p)$, and $f_{i}(x, p)$ respectively. Denote $\partial f_{i}(x, p) / \partial x_{j}$ by $f_{i j}(x, p)$.

Theorem 4. Let $G$ be a locally compact group of transformation of class $C^{1}$ of a manifold $M$ of class $C^{1}$. Suppose $G$ acts on $M$ in such a way that the identity is the only element whose set of fix points contains interior points. Then $G$ is a Lie group.

Proof. Take a sufficiently small neighborhood $V$ of $e$ and open sets $Q_{1}, Q_{2}$ with $\bar{V}\left(\bar{Q}_{1}\right) \subset Q_{2}, V\left(\bar{Q}_{2}\right) \subset Q$. Denote by $|p-q|(p, q \in Q)$ the euclidean metric in $Q$ and define

$$
\rho(x, y)=\max _{\tau \in Q 1}|x(p)-y(p)|
$$

for $x, y \in \bar{V}$. It is easily verified that $\rho$ is a metric in $\bar{V} .^{13)}$

As $x(p)$ is a transformation of class $C^{1}$, we have

$$
x_{i}(p)-x_{i}(q)=\sum\left(p_{j}-q_{j}\right) \int_{0}^{1} f_{i j}(x ; q+t(p-q)) d t
$$

for every $x \in \bar{V}$ and $p, q \in \bar{Q}_{2}$.

Since it is known ${ }^{14)}$ that $f_{i j}(x, p)$ is continuous in $\bar{V} \times \bar{Q}, f_{i j}(x, p)$ is near to $\delta_{j}^{i}$ uniformly in $p$, if $x$ is sufficiently near to $e$. From this follows

$$
|x(p)-x(q)-(p-q)| \leqq M(|x|)|p-q|,
$$

12) Cf. [6].

13) Theorem 3 of [6].

14) Cf. [5]. 
where $M(\lambda)^{15)}$ is a function such that $M(\lambda) \rightarrow 0$ as $\lambda \rightarrow 0$, and $|x|=\rho(x, e)$.

If we put $p=y(q)$

(18) $|(x y(q)-q)-\{(x(q)-q)+(y(q)-q)\}| \leqq M(|x|)|y(q)-q| \leqq M(|x|)|y|$. If we put $p=y(\boldsymbol{r}), q=w(\boldsymbol{r})$ in (17), it follows that

whence

$$
|x y(\boldsymbol{r})-x w(\boldsymbol{r})-(y(\boldsymbol{r})-w(\boldsymbol{r}))| \leqq M(|x|)|y(\boldsymbol{r})-w(\boldsymbol{r})|,
$$

$$
\rho(x y, x w) \leqq K \rho(y, w) .
$$

From (19) follows, putting $w=e$,

$$
|x y| \leqq|x|+K|y| \text {. }
$$

If we assume that $x, x^{2}, \ldots, x^{m} \in \bar{V}$ and that for $q \in \bar{Q}_{1}$

$$
\left|\left(x^{m-1}(q)-q\right)-(m-1)(x(q)-q)\right| \leqq M((m-1)|x|)|x(q)-q|,
$$

it follows from (18) that

$$
\begin{aligned}
& \mid\left(x^{m}(q)-q\right)-m((x(q)-q) \mid \\
& =\left|\left(x^{m-1} \cdot x(q)-q\right)-\{(x(q)-q)+(m-1)(x(q)-q)\}\right| \\
& \leqq\left\{\left(x^{m-1} \cdot x(q)-q\right)-\left\{(x(q)-q)+\left(x^{m-1}(q)-q\right)\right\} \mid\right. \\
& +\left|\left(x^{m-1}(q)-q\right)-(m-1)(x(q)-q)\right| \\
& \leqq M\left(\left|x^{m-1}\right|\right)|x(q)-q|+M((m-1)|x|)(m-1)|x(q)-q| \\
& \leqq M(m|x|) m|x(q)-q| \text {. }
\end{aligned}
$$

Thus if $x, x^{2}, \ldots, x^{m} \in \bar{V}$

$$
\left|\left(x^{m}(q)-q\right)-m(x(q)-q)\right| \leqq M(m|x|) m|x(q)-q| \leqq M(m|x|) m|x| .
$$

$$
K_{2} m|x| \leqq\left|x^{m}\right| \leqq K_{1} m|x| \text { (when } V \text { is sufficiently small). }
$$

From (19) and (22) follows ${ }^{16)}$

$$
\rho(y w, x w) \leqq K \rho(y, x) .
$$

By (19), (23)

$$
\rho\left(x^{m}, y^{m}\right) \leqq \sum_{k=0}^{m} \rho\left(x^{m-k} y^{k}, x^{m-(k+1)} y^{k+1}\right) \leqq K^{2} m \rho(x, y),
$$

By (22), $G$ has property (S). Let $V_{1}$ be a neighborhood of $e$ in which the above inequalities stands. Take a regular neighborhood $U \subset V_{1}$. We shall show that $G$ satisfies the conditions of Theorem 2 with $U$. If $x, y \in \Phi_{n+s}^{\bar{U}}$, it follows that

$$
\begin{aligned}
& \left|\left(x^{1 / 2^{n}} y^{1 / 2^{n}}(q)-q\right)-\left\{\left(x^{1 / 2^{n}}(q)-q\right)+\left(y^{1 / 2^{n}}(q)-q\right)\right\}\right| \leqq M\left(1 / 2^{n}|x|\right)\left(1 / 2^{n}\right)|y|((18)), \\
& \left|-\left(\left(x^{1 / 2^{n+s}} y^{1 / 2^{n+s}}\right)^{2^{s}}(q)-q\right)+2^{s}\left(x^{1 / 2^{n+s}} y^{1 / 2^{n+s}}(q)-q\right)\right| \\
& \quad \leqq M\left(\left(1 / 2^{n}\right)|x|+\left(1 / 2^{n}\right)|y|\right)\left(1 / 2^{n}\right)\{|x|+|y|\}
\end{aligned}
$$

15. It goes without saying that $M(\lambda)$ varies from inequality to inequality. But since only finite numbers of $M(\lambda)$ are used, we can use a unique $M(\lambda)$.

16) Consult the beginning of $\S 2$ of [4]. 


$$
\begin{aligned}
& \left|-2^{s}\left\{x^{1 / 2^{l i+s}} y^{1 / 2^{2+s}}(q)-q\right\}+2^{s}\left\{\left(x^{1 / 2^{n+s}}(q)-q\right)+\left(y^{1 / 2^{n+s}}(q)-q\right)\right\}\right| \\
& \leqq M\left(1 / 2^{n+s}|x|\right)\left(1 / 2^{n}\right)\lfloor y \mid \quad((18)), \\
& \left|\left\{\left(x^{1 / 2^{2 x+s}}\right)^{2^{s}}(q)-q\right\}-2^{s}\left(x^{1 / 2^{n+s}}(q)-q\right)\right| \leqq M\left(\left(1 / 2^{n}\right)|x|\right)\left(1 / 2^{n}\right)|x| \quad((21),(22)), \\
& \left|\left\{\left(y^{1 / 2^{n+s}}\right)^{2^{s}}(q)-q\right\}-2^{s}\left(y^{1 / 2^{n+s}}(q)-q\right)\right| \leqq M\left(\left(1 / 2^{n}\right)|y|\right)\left(1 / 2^{n}\right)|y| \text {. }
\end{aligned}
$$

Summing up these five inequalities, we get

(25) $\left|x^{1 / 2^{n}} y^{1 / 2^{n}}(q)-\left(x^{1 / 2^{n+s}} y^{1 / 2^{n+s}}\right)^{2^{s}}(q)\right| \leqq M\left(\left(1 / 2^{n}\right)|x|+\left(1 / 2^{n}\right)|y|\right)\left(1 / 2^{n}\right)(|x|+|y|)$.

By (24) and (25)

$$
\begin{aligned}
\rho\left(\left(x^{1 / 2^{n}} y^{1 / 2^{n}}\right)^{2^{n}}\right. & \left.,\left(x^{1 / 2^{n+s}} y^{1 / 2^{n+s}}\right)^{2^{n+s}}\right) \leqq 2^{n} K^{2} \rho\left(x^{1 / 2^{n}} y^{1 / 2^{n}},\left(x^{1 / 2^{n+s}} y^{1 / 2^{n+s}}\right)^{2^{s}}\right) \\
& \leqq M\left(\left(1 / 2^{n}\right)|x|+\left(1 / 2^{n}\right)|y|\right)(|x|+|y|) \leqq M\left(\left(1 / 2^{n}\right) \widetilde{K}\right) \widetilde{K}
\end{aligned}
$$

for $x, y \in \Phi_{n+s}^{\vec{c}}$. Thus $G$ satisfies the condition (C) of Theorem 2, and by the remark to Theorem $2 G$ must be a Lie group.

\section{REFERENCE}

[1] Garret Birkhoff, Analytical groups, Trans. Am. Math. Soc. Vol. 43 (1943). 61-101.

[2] Gleason, A., On the structure of locally compact groups, Proc. of Nat. Acad. Vol. 35 (1949), pp. 384-386.

[3] Iwasawa, K., On some types of topological groups, Ann. of Math. Vol. 50 (1949), pp. 507-558.

[4] Kuranishi, M., On euclidean local groups satisfying certain conditions, to appear in Bull. Amer. Math. Soc.

[5] Montgomery, D., Topological groups of differentiable transformations, Ann. of Math., Vol. 46 (1945), pp. 382-387.

[6] Montgomery, D., Locally compact groups of differentiable transformations, Ann. of Math., Vol, 47 (1946), pp. 639-653.

[7] Montgomery, D., Connected one-dimensional group, Ann. of Math. Vol. 49 (1948), pp. 110-117.

[8] Pontrjagin, L., Topological groups, 1936, Princeton.

[9] Smith, P., Foundations of the theory of Lie groups with real parameter. Ann. of Math. Vol. 44 (1943), pp. 481-513.

[10] Weil, A., Sur les espaces à structure uniform ct sur la topologie générale, Actualitiés Scien. et Industrielles 55, 1938. Hermann, Paris. 